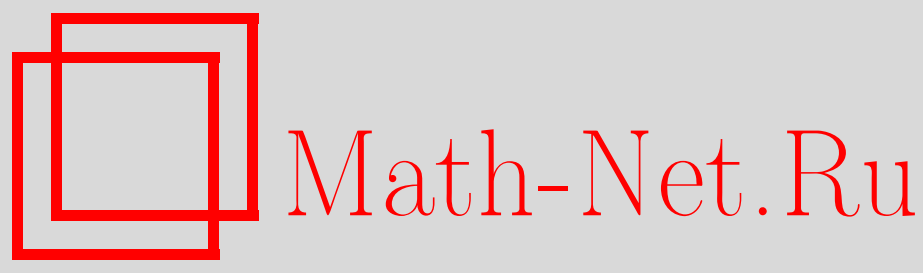

Н. Р. Хуснутдинов, Р. Н. Кашапов, Эффект Казимира для совокупности параллельных проводящих поверхностей, ТМФ, 2015, том 183, номер 1, 51-61

DOI: https://doi.org/10.4213/tmf8788

Использование Общероссийского математического портала Math-Net.Ru подразумевает, что вы прочитали и согласны с пользовательским соглашением http://www . mathnet.ru/rus/agreement

Параметры загрузки:

IP: 54.162 .85 .209

26 апреля 2023 г., 09:51:23

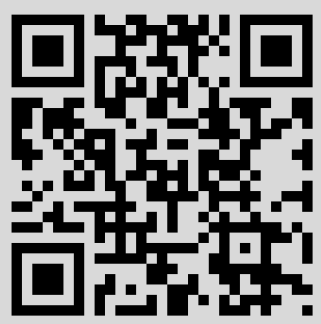




\title{
ЭФФЕКТ КАЗИМИРА ДЛЯ СОВОКУПНОСТИ ПАРАЛЛЕЛЬНЫХ ПРОВОДЯЩИХ ПОВЕРХНОСТЕЙ
}

\begin{abstract}
Рассматривается энергия Казимира для системы проводящих параллельных плоскостей, обладающих постоянной поверхностной проводимостью. Получено общее выражение для энергии Казимира системы двух, трех и четырех плоскостей. В случае одинакового расстояния между плоскостями энергия обратно пропорциональна кубу расстояния между плоскостями и при малой проводимости не зависит от постоянной Планка и скорости света. Для системы идеально проводящих плоскостей энергия Казимира является суммой энергий Казимира пар соседних плоскостей.
\end{abstract}

Ключевые слова: поляризация вакуума, эффект Казимира, дзета-функция, квантовая теория поля.

DOI: $10.4213 / \operatorname{tmf} 8788$

\section{1. ВВЕДЕНИЕ}

За 60 лет, прошедших с момента выхода замечательной работы Казимира [1], произошел значительный прогресс как в экспериментальном обосновании, так и в теоретическом описании эффекта, получившего название эффект Казимира. В настоящее время имеется ряд обзоров [2]-[5] и книг [6], [7], в которых с различных точек зрения рассматривается это явление. Взаимодействие между телами на малых расстояниях, когда запаздыванием электромагнитного взаимодействия можно пренебречь, обычно называют силами ван дер Ваальса. На больших расстояниях эффект запаздывания является существенным, и соответствующие силы называют силами Казимира. В частном случае взаимодействия атомов потенциал притяжения на малых расстояниях обратно пропорционален шестой степени расстояния, тогда как на больших расстояниях он падает, как седьмая степень расстояния. Параметр, разделяющий малые и большие расстояния, определяется моделью, описывающей поверхность.

Существуют два основных подхода к описанию сил Казимира. Первый, восходящий к Казимиру, рассматривает силы как результат влияния границ тела на спектр вакуумных флуктуаций электромагнитного поля. Основной энергией, приводящей

* Казанский федеральный университет, Казань, Россия.

E-mail: nail.khusnutdinov@gmail.com, kashapov.rashid@gmail.com 
к взаимодействию между телами, является энергия нулевых (вакуумных) колебаний поля. Такой подход иногда называют методом суммирования спектра. Второй подход основан на рассмотрении флуктуаций межмолекулярных сил. Общая теория молекулярных сил ван дер Ваальса-Казимира была построена Лифшицем в 1955 г. [8]. Метод, основанный на корреляционных функциях флуктуаций электромагнитного поля, был развит в работах [9], [10].

Метод суммирования спектра приводит к бесконечным выражениям, поскольку энергия нулевых колебаний, определяющаяся как сумма по всему спектру1),

$$
\mathcal{E}=\frac{1}{2} \sum_{(n)} \omega_{(n)}
$$

расходится. Для придания смысла этому выражению необходимо его регуляризовать. При помодовой регуляризации под знак суммы вносят обрезающий множитель вида $e^{-\epsilon \omega_{n}}$ с последующим взятием предела $\epsilon \rightarrow 0$. Другой вид регуляризации, называемый дзета-регуляризацией, основан на рассмотрении мероморфной функции переменной $s$

$$
\mathcal{E}(s)=\frac{1}{2} M^{2 s} \sum_{(n)} \omega_{(n)}^{1-2 s}=\frac{1}{2} M^{2 s} \zeta_{\widehat{L}}(1-2 s),
$$

где $\zeta_{\widehat{L}}(s)$ является обобщенной дзета-функцией оператора $\widehat{L}$ с собственными значениями $\omega_{(n)}$ и с соответствующими граничными условиями. Величина $M$ с размерностью массы введена для того, чтобы функция $\mathcal{E}(s)$ имела размерность энергии независимо от $s$. Для достаточно больших значений Re $s$ ряд сходится, и можно показать, что обобщенная дзета-функция $\zeta_{\widehat{L}}(s)$ имеет конечное число полюсов, количество и положение которых зависит как от граничных условий, так и от размерности задачи. В пределе $s \rightarrow 0$ энергия нулевых колебаний расходится, и расходимость выделяется в виде полюсов. После соответствующей перенормировки получается конечное выражение для энергии Казимира. Впервые дзета-регуляризация была введена в работе Доукера и Критчли [11] и независимо Хокингом в статье [12]. Применительно к эффекту Казимира дзета-регуляризация была рассмотрена в работе [13]. Применение дзета-функции в различных областях физики можно найти, например, в книге [14].

В большинстве случаев найти спектр в явном виде не удается. Чтобы обойти эту проблему, в работе [15] (см. также полное изложение метода в книге [7]) было предложено интегральное представление дзета-функции, не требующее получения спектра в явном виде. Разделим мультииндекс $(n)$ на две части: $(n)=(k), j$, где индекс $j=1,2, \ldots$ нумерует решения граничной задачи

$$
\Psi\left(\omega_{(k), j}\right)=0 .
$$

Используя принцип аргумента, можно представить сумму по индексу $j$ в виде контурного интеграла и трансформировать контур на всю мнимую ось. Интеграл от $-m$ до $+m$ равен нулю, а интегралы по интервалам $(-\infty,-m)$ и $(+m,+\infty)$ отличаются только фазой. В итоге получаем выражение для регуляризованной энергии

$$
\mathcal{E}(s)=-M^{2 s} \frac{\cos \pi s}{2 \pi} \sum_{(k)} \int_{m}^{\infty} d \lambda\left(\lambda^{2}-m^{2}\right)^{1 / 2-s} \frac{\partial}{\partial \lambda} \ln \Psi(i \lambda)
$$

\footnotetext{
1) Мы используем систему единиц $\hbar=c=1$, в которой размерности частоты, волнового вектора, массы и энергии одинаковы и равны обратным метрам.
} 
в случае скалярного массивного поля, удовлетворяющего уравнению Клейна-Гордона-Фока. Таким образом, для применения этого метода необходимо получить в явном виде функцию $\Psi$, определяющую спектр энергий электромагнитного поля.

Как отмечалось в трудах [7], [16], в диссипативной среде частоты становятся комплексными, и формула (1) неприменима. В то же время авторы этих работ показывают, что формула (2), аналогичная формуле Лифшица, применима и в этом случае. По этой причине мы будем использовать формулу (2) для вычисления энергии Казимира. В недавней работе [17] было показано, что эта формула содержит в себе сумму вкладов поверхностных плазмонов и состояний рассеяния.

В пределе $s \rightarrow 0$ полученное выражение расходится, и необходимо произвести дополнительные перенормировки. Известно (см., например, книгу [7]), что в указанном пределе расходимости имеют характер полюсов и полностью определяются коэффициентами теплового ядра оператора $\widehat{L}$. Стандартная степенная асимптотика теплового ядра приводит к простым полюсам дзета-функции, тогда как нетривиальные граничные условия могут приводить к логарифмической асимптотике теплового ядра и, как следствие, к полюсам второго порядка дзета-функции [18], [19].

В настоящей работе мы проведем подробный анализ энергии Казимира для набора параллельных проводящих плоскостей в рамках дзета-регуляризации. Физическим объектом, к которому эти вычисления имеют непосредственное отношение, является графен - монослой атомов углерода [20]. Графен - это проводник, проводимость которого постоянна, $\sigma=e^{2} / 4$ (e- заряд электрона), вплоть до энергий $\mathcal{E}_{\mathrm{t}} \sim 3$ эВ (соответствующая частота $\omega_{\mathrm{t}} \sim 4.5$ ПГц и длина волны $\lambda_{\mathrm{t}} \sim 413 \mathrm{Hм}$ ). Это было предсказано теоретически [21] и подтверждено экспериментально в рабо$\operatorname{таx~[22],~[23].~}$

В настоящей работе мы рассмотрим следующую модель проводимости поверхностей: предположим, что проводимость является постоянной не только до энергий порядка 3 эВ, но и на любой частоте. Теория эффекта Казимира для системы графен-металл была разработана в работах [24]-[26] на основе вычисления поляризационного оператора двумерных дираковских фермионов при конечной температуре.

Необходимо подчеркнуть, что размерность проводимости для двумерных поверхностей совпадает с размерностью скорости. По этой причине в релятивистской теории возникает безразмерный параметр $\eta=2 \pi \sigma / c$, который для графена равен $\pi e^{2} / 2 \hbar c=\pi \alpha / 2 \approx 0.0115$, где $\alpha-$ постоянная тонкой структуры.

\section{2. ОБЩИЕ СООТНОШЕНИЯ}

В случае плоской симметрии электромагнитное поле разлагается на две составляющие, которые обычно называют ТЕ- и ТМ-модами. Расположим в вакууме пластины параллельно плоскости $(x, y)$. Тогда ТЕ-мода имеет равную нулю нормальную компоненту электрического поля, $E_{z}=0$, а ТМ-мода имеет равную нулю нормальную магнитную компоненту, $H_{z}=0$. Полная энергия представляется суммой вкладов ТЕ-и ТМ-поляризаций. Вследствие симметрии представим поле в следующем виде:

$$
\mathbf{E}=\mathbf{e}(z) e^{i k_{x} x+i k_{y} y-i \omega t}, \quad \mathbf{H}=\mathbf{h}(z) e^{i k_{x} x+i k_{y} y-i \omega t} .
$$

Уравнения Максвелла вне поверхностей не содержат токов и зарядов и позволяют выразить все компоненты поля через одну: 
в случае ТЕ-моды $\left(e_{z}=0\right)$

$$
\begin{array}{rlrl}
e_{x} & =-\frac{\omega k_{y}}{c k_{\perp}^{2}} h_{z}, & h_{x} & =\frac{i k_{x}}{k_{\perp}^{2}} h_{z}^{\prime}, \\
e_{y}=\frac{\omega k_{x}}{c k_{\perp}^{2}} h_{z}, & h_{y} & =\frac{i k_{y}}{k_{\perp}^{2}} h_{z}^{\prime}, \\
h_{z}^{\prime \prime} & =k^{2} h_{z}
\end{array}
$$

в случае ТМ-моды $\left(h_{z}=0\right)$

$$
\begin{array}{rlrl}
e_{x} & =\frac{i k_{x}}{k_{\perp}^{2}} e_{z}^{\prime}, & h_{x}=\frac{\omega k_{y}}{c k_{\perp}^{2}} e_{z}, \\
e_{y} & =\frac{i k_{y}}{k_{\perp}^{2}} e_{z}^{\prime}, & h_{y}=-\frac{\omega k_{x}}{c k_{\perp}^{2}} e_{z}, \\
e_{z}^{\prime \prime} & =k^{2} e_{z} .
\end{array}
$$

Здесь $k_{\perp}^{2}=k_{x}^{2}+k_{y}^{2}, k^{2}=k_{\perp}^{2}-\omega^{2}$ и штрих означает производную по $z$.

Вся информация о поверхностях закодирована в граничных условиях, которые имеют следующий вид:

$$
\begin{aligned}
\left(\mathbf{B}_{1}-\mathbf{B}_{2}\right)_{S} \cdot \mathbf{n}_{1 \rightarrow 2}=0, & \left(\mathbf{D}_{1}-\mathbf{D}_{2}\right)_{S} \cdot \mathbf{n}_{1 \rightarrow 2}=-4 \pi \rho_{\mathrm{s}}, \\
\left(\mathbf{E}_{1}-\mathbf{E}_{2}\right)_{S} \times \mathbf{n}_{1 \rightarrow 2}=0, & \left(\mathbf{H}_{1}-\mathbf{H}_{2}\right)_{S} \times \mathbf{n}_{1 \rightarrow 2}=\frac{4 \pi}{c} \mathbf{j}_{\mathrm{s}},
\end{aligned}
$$

где $\rho_{\mathrm{s}}$ представляет собой флуктуацию поверхностного заряда, а $\mathbf{j}_{\mathrm{s}}$ является поверхностной плотностью тока, связанной с электрическим полем линейным соотношением $\mathbf{j}_{\mathrm{s}}=\sigma \mathbf{E}$.

Для плоскости, расположенной в точке $z=a$, граничные условия выглядят следующим образом:

$$
\begin{array}{ll}
\text { ТЕ-мода : } & {\left[h_{z}\right]_{r}=0, \quad\left[h_{z}^{\prime}\right]_{r}=\left.4 \pi i \sigma \omega h_{z}\right|_{r},} \\
\text { ТМ-мода : } & {\left[e_{z}^{\prime}\right]_{r}=0, \quad\left[e_{z}\right]_{r}=-\left.\frac{4 \pi i \sigma}{\omega} e_{z}^{\prime}\right|_{r},}
\end{array}
$$

где $[f(z)]_{r}=f(r-0)-f(r+0)$.

Таким образом, для каждой плоскости мы имеем два уравнения для каждой моды. Для $n$ параллельных плоскостей имеются $2 n$ уравнений. Количество интервалов между плоскостями равно $n-1$, и в каждом таком зазоре решение содержит две константы, общее число констант равно $2 n-2$. Вне плоскостей имеются два полупространства, в каждом из которых решение определяется одной константой при условии равенства нулю поля на бесконечности. Всего получаем ровно $2 n$ констант, удовлетворяющих $2 n$ уравнениям. Условие равенства нулю главного определителя системы позволяет получить в явном виде функцию $\Psi$, необходимую для вычисления энергии по формуле (2), которая в случае электромагнитного поля имеет вид суммы $\mathcal{E}(s)=\mathcal{E}_{\mathrm{TE}}(s)+\mathcal{E}_{\text {тм }}(s)$ вкладов ТЕ- и ТМ-поляризаций; здесь

$$
\mathcal{E}_{k}(s)=-M^{2 s} \frac{\cos \pi s}{2 \pi} \iint \frac{d^{2} k}{(2 \pi)^{2}} \int_{0}^{\infty} d \lambda \lambda^{1-2 s} \frac{\partial}{\partial \lambda} \ln \Psi_{k}(i \lambda), \quad k=\text { TE, TM. }
$$

Рассмотрим некоторые частные случаи. 


\section{3. ПОСТОЯННАЯ ПРОВОДИМОСТЬ, $\sigma=$ const}

3.1. Две плоскости. Рассмотрим классический случай двух параллельных плоскостей. Расположим плоскости в точках $z=0, d$. Из граничных условий получаем

$$
\begin{aligned}
\Psi_{\mathrm{TE}}(i \lambda) & =(\kappa+\eta \lambda)^{2}-\eta^{2} \lambda^{2} e^{-2 d \kappa}, \\
\Psi_{\mathrm{TM}}(i \lambda) & =\frac{\kappa^{2}}{\lambda^{2}}\left\{(\eta \kappa+\lambda)^{2}-\eta^{2} \kappa^{2} e^{-2 d \kappa}\right\},
\end{aligned}
$$

где $\kappa=\sqrt{\lambda^{2}+k_{\perp}^{2}}$. Для получения конечного в пределе $s \rightarrow 0$ выражения вычтем из регуляризованной энергии ее значение в пределе $d \rightarrow \infty$. Это отвечает разумному физическому требованию равенства нулю энергии при бесконечном удалении пластин друг от друга. После такого вычитания можно положить $s=0$, и мы получаем следующие выражения для энергии в случае двух пластин:

$$
\begin{aligned}
& \mathcal{E}_{\mathrm{TE}}^{(2)}=\frac{1}{8 \pi^{3}} \iint d^{2} k \int_{0}^{\infty} d \lambda \ln \left(1-\frac{\eta^{2} \lambda^{2} e^{-2 d \kappa}}{(\kappa+\eta \lambda)^{2}}\right), \\
& \mathcal{E}_{\mathrm{TM}}^{(2)}=\frac{1}{8 \pi^{3}} \iint d^{2} k \int_{0}^{\infty} d \lambda \ln \left(1-\frac{\eta^{2} \kappa^{2} e^{-2 d \kappa}}{(\eta \kappa+\lambda)^{2}}\right) .
\end{aligned}
$$

Переходя к сферическим координатам и полагая $\kappa=y / d$, получаем

$$
\mathcal{E}^{(2)}=\frac{Q^{(2)}(\eta)}{d^{3}}, \quad Q^{(2)}=Q_{\mathrm{TE}}^{(2)}+Q_{\mathrm{TM}}^{(2)},
$$

где

$$
\begin{aligned}
& Q_{\mathrm{TE}}^{(2)}(\eta)=\frac{1}{32 \pi^{2}} \int_{0}^{\infty} y^{2} d y \int_{0}^{1} d x \ln \left(1-\frac{\eta^{2} x^{2} e^{-y}}{(1+\eta x)^{2}}\right), \\
& Q_{\mathrm{TM}}^{(2)}(\eta)=\frac{1}{32 \pi^{2}} \int_{0}^{\infty} y^{2} d y \int_{0}^{1} d x \ln \left(1-\frac{\eta^{2} e^{-y}}{(x+\eta)^{2}}\right)
\end{aligned}
$$

здесь $x=\cos \theta$. Таким образом, энергия Казимира обратно пропорциональна кубу расстояния для любых расстояний между пластинами.

В пределе идеальной проводимости $\eta \rightarrow \infty$ получаем

$$
\mathcal{E}^{(2)}=-\frac{\pi^{2}}{720 d^{3}}
$$

в полном согласии с результатом Казимира. В обратном пределе малой проводимости $\eta \rightarrow 0$ основной линейный вклад происходит от ТМ-моды:

$$
\mathcal{E}^{(2)}=-\frac{a_{2} \eta}{2 \pi d^{3}}=-\frac{a_{2} \sigma}{d^{3}},
$$

где

$$
\begin{aligned}
a_{2} & =-\frac{1}{16 \pi} \int_{0}^{\infty} y^{2} d y \int_{0}^{\infty} d x \ln \left(1-\frac{e^{-y}}{(1+x)^{2}}\right)= \\
& =\frac{1}{2 \pi}\left(-\frac{\pi^{2}}{6}-\frac{\pi^{4}}{360}+4 \ln 2-\frac{1}{2} \zeta_{\mathrm{R}}(3)\right) \approx 0.0407509 \ldots
\end{aligned}
$$


и $\zeta_{\mathrm{R}}(a)$ является дзета-функцией Римана. ТЕ-мода дает квадратичный по проводимости вклад. Для проводимости, равной проводимости графена, получаем

$$
\mathcal{E}^{(2)}=-\frac{e^{2} b}{32 \pi d^{3}},
$$

где $b=8 \pi a_{2} \approx 1.0241 \ldots$. Следует отметить, что полученное выражение в размерных единицах имеет точно такой же вид, и энергия Казимира не зависит от постоянной Планка и скорости света. Этот результат был получен в работах [27], [28] на основе различных вычислений.

3.2. Три плоскости. Применим метод, изложенный выше, к трем параллельным пластинам, расположенным в точках $d_{1}<d_{2}<d_{3}$. Обозначим через $d_{i k}$ расстояние между $i$-й и $k$-й плоскостями. Чтобы получить конечное выражение, вычтем из энергии значение, полученное последовательным взятием пределов $d_{3} \rightarrow \infty$ и $d_{2} \rightarrow \infty$. Переходя к сферическим координатам, выводим следующие формулы для энергий:

$$
\begin{aligned}
& \mathcal{E}_{\mathrm{TE}}^{(3)}=\frac{1}{32 \pi^{2}} \int_{0}^{\infty} \kappa^{2} d \kappa \int_{0}^{1} d x \ln \left(1-\frac{\eta^{2} x^{2}}{(1+\eta x)^{2}}\left\{e^{-d_{21} \kappa}+e^{-d_{32} \kappa}+\frac{1-\eta x}{1+\eta x} e^{-d_{31} \kappa}\right\}\right), \\
& \mathcal{E}_{\mathrm{TM}}^{(3)}=\frac{1}{32 \pi^{2}} \int_{0}^{\infty} \kappa^{2} d \kappa \int_{0}^{1} d x \ln \left(1-\frac{\eta^{2}}{(\eta+x)^{2}}\left\{e^{-d_{21} \kappa}+e^{-d_{32} \kappa}+\frac{x-\eta}{x+\eta} e^{-d_{31} \kappa}\right\}\right) .
\end{aligned}
$$

При удалении первой или третьей плоскости на бесконечность мы возвращаемся к результату предыдущего пункта - энергии Казимира между двумя плоскостями.

Полученное выше выражение не представляет собой линейную суперпозицию энергий между плоскостями. Действительно, выделим в выражении под логарифмом, например, для ТМ-моды произведение выражений для пар первой-второй и второй-третьей плоскостей:

$$
\left(1-\frac{\eta^{2} e^{-d_{21} \kappa}}{(x+\eta)^{2}}\right)\left(1-\frac{\eta^{2} e^{-d_{32} \kappa}}{(x+\eta)^{2}}\right)-\frac{\eta^{2} x^{2} e^{-d_{31} \kappa}}{(x+\eta)^{4}} .
$$

Используя это соотношение, представим энергию в следующем виде:

$$
\mathcal{E}_{\mathrm{TM}}^{(3)}=\frac{Q_{\mathrm{TM}}^{(2)}(\eta)}{d_{21}^{3}}+\frac{Q_{\mathrm{TM}}^{(2)}(\eta)}{d_{32}^{3}}+\widetilde{\mathcal{E}}_{\mathrm{TM}}^{(3)}\left(d_{21}, d_{32}, d_{31}\right),
$$

где

$$
\widetilde{\mathcal{E}}_{\mathrm{TM}}^{(3)}=\frac{1}{32 \pi^{2}} \int_{0}^{\infty} \kappa^{2} d \kappa \int_{0}^{1} d x \ln \left(1-\frac{\eta^{2} x^{2} e^{-d_{31} \kappa}}{\left((x+\eta)^{2}-\eta^{2} e^{-d_{21} \kappa}\right)\left((x+\eta)^{2}-\eta^{2} e^{-d_{32} \kappa}\right)}\right)
$$

зависит от расстояний $d_{21}, d_{32}, d_{31}$ между любыми двумя плоскостями. Аналогично для ТЕ-моды имеем

$$
\mathcal{E}_{\mathrm{TE}}^{(3)}=\frac{Q_{\mathrm{TE}}^{(2)}(\eta)}{d_{21}^{3}}+\frac{Q_{\mathrm{TE}}^{(2)}(\eta)}{d_{32}^{3}}+\widetilde{\mathcal{E}}_{\mathrm{TE}}^{(3)}\left(d_{21}, d_{32}, d_{31}\right),
$$

где

$\widetilde{\mathcal{E}}_{\mathrm{TE}}^{(3)}=\frac{1}{32 \pi^{2}} \int_{0}^{\infty} \kappa^{2} d \kappa \int_{0}^{1} d x \ln \left(1-\frac{\eta^{2} x^{2} e^{-d_{31} \kappa}}{\left((1+x \eta)^{2}-\eta^{2} x^{2} e^{-d_{21} \kappa}\right)\left((1+x \eta)^{2}-\eta^{2} x^{2} e^{-d_{32} \kappa}\right)}\right)$. 
Таким образом, суммарная энергия Казимира

$$
\mathcal{E}^{(3)}=\mathcal{E}^{(2)}\left(d_{21}\right)+\mathcal{E}^{(2)}\left(d_{32}\right)+\widetilde{\mathcal{E}}^{(3)}\left(d_{21}, d_{32}, d_{31}\right)
$$

представляет собой сумму энергий Казимира для пар первой--второй и второй-третьей плоскостей и вклада, нелинейно зависящего от расстояний между всеми плоскостями. В пределе идеальной проводимости $\eta \rightarrow \infty$ последнее слагаемое равно нулю, и энергия Казимира представляет собой сумму энергий Казимира для каждой пары соседних плоскостей:

$$
\mathcal{E}^{(3)}=\mathcal{E}_{\mathrm{c}}^{(2)}\left(d_{21}\right)+\mathcal{E}_{\mathrm{c}}^{(2)}\left(d_{32}\right) .
$$

В случае равных расстояний между плоскостями, $d_{21}=d_{32}=d$, зависимость от расстояния между плоскостями имеет простой вид

$$
\mathcal{E}^{(3)}=\frac{Q^{(3)}(\eta)}{d^{3}}, \quad Q^{(3)}=Q_{\mathrm{TE}}^{(3)}+Q_{\mathrm{TM}}^{(3)},
$$

где

$$
\begin{aligned}
& Q_{\mathrm{TE}}^{(3)}=\frac{1}{32 \pi^{2}} \int_{0}^{\infty} y^{2} d y \int_{0}^{1} d x \ln \left(1-\frac{\eta^{2} x^{2} e^{-y}}{(1+\eta x)^{2}}\left\{2+\frac{1-\eta x}{1+\eta x} e^{-y}\right\}\right) \\
& Q_{\mathrm{TM}}^{(3)}=\frac{1}{32 \pi^{2}} \int_{0}^{\infty} y^{2} d y \int_{0}^{1} d x \ln \left(1-\frac{\eta^{2} e^{-y}}{(x+\eta)^{2}}\left\{2+\frac{x-\eta}{x+\eta} e^{-y}\right\}\right)
\end{aligned}
$$

Для малой проводимости $\eta \ll 1$ получаем

$$
\mathcal{E}^{(3)}=-\frac{a_{3} \eta}{2 \pi d^{3}}=-\frac{a_{3} \sigma}{d^{3}},
$$

где

$$
a_{3}=-\frac{1}{16 \pi} \int_{0}^{\infty} y^{2} d y \int_{0}^{\infty} d x \ln \left(1-\frac{e^{-y}}{(x+1)^{2}}\left\{2+\frac{x-1}{x+1} e^{-y}\right\}\right)=0.0832892 \ldots
$$

Рассмотрим количественно влияние третьей плоскости на энергию системы двух плоскостей. Для этого нормируем все расстояния на расстояние $d=d_{21}$ между первой и второй плоскостями и выделим часть (3), соответствующую двум плоскостям. Получаем следующее выражение:

$$
\mathcal{E}^{(3)}=\mathcal{E}^{(2)}(d) \Delta\left(\eta, q_{32}\right), \quad \Delta\left(\eta, q_{32}\right)=\frac{Q_{\mathrm{TE}}^{(3)}\left(\eta, q_{32}\right)+Q_{\mathrm{TM}}^{(3)}\left(\eta, q_{32}\right)}{Q_{\mathrm{TE}}^{(2)}(\eta)+Q_{\mathrm{TM}}^{(2)}(\eta)},
$$

где

$$
\begin{aligned}
& Q_{\mathrm{TE}}^{(3)}=\frac{1}{32 \pi^{2}} \int_{0}^{\infty} y^{2} d y \int_{0}^{1} d x \ln \left(1-\frac{\eta^{2} x^{2}}{(1+\eta x)^{2}}\left\{e^{-y}+e^{-q_{32} y}+\frac{1-\eta x}{1+\eta x} e^{-\left(1+q_{32}\right) y}\right\}\right), \\
& Q_{\mathrm{TM}}^{(3)}=\frac{1}{32 \pi^{2}} \int_{0}^{\infty} y^{2} d y \int_{0}^{1} d x \ln \left(1-\frac{\eta^{2}}{(x+\eta)^{2}}\left\{e^{-y}+e^{-q_{32} y}+\frac{x-\eta}{x+\eta} e^{-\left(1+q_{32}\right) y}\right\}\right)
\end{aligned}
$$

и $q_{32}=d_{32} / d$. 


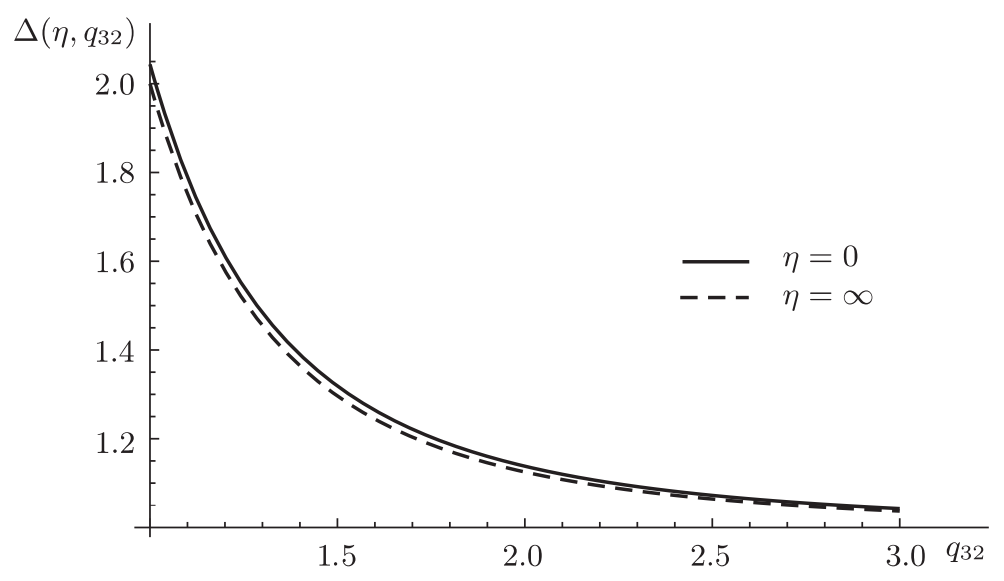

Рис. 1. Зависимость величины $\Delta$ от безразмерного расстояния $q_{32}=d_{32} / d$ $\left(d-\right.$ расстояние между первой и второй плоскостями, $d_{32}-$ расстояние между второй и третьей плоскостями) для двух предельных значений проводимости $\eta=0$ и $\eta=\infty$ (идеальный случай).

Численный расчет показывает, что величина $\Delta$ слабо зависит от проводимости, если третья плоскость находится на расстоянии, большем расстояния между первой и второй плоскостями, $d_{3}-d_{2} \geqslant d$. Если же третья плоскость находится ближе, то ситуация меняется, и доминирующей становится энергия между второй и третьей плоскостями, а первая плоскость играет роль дополнительной, третьей. На рис. 1 изображены зависимости величины $\Delta$ для двух предельных значений проводимости $\eta=0$ и $\eta=\infty$ (идеальный случай). Видно, что кривые слабо отличаются друг от друга в указанном интервале расстояний.

Расположим третью плоскость достаточно далеко от первых двух, т. е. положим $q_{32} \gg 1$. В этом случае

$$
\Delta \approx 1+\frac{A(\eta)}{q_{32}^{3}}
$$

и

$$
\mathcal{E}^{(3)}=\mathcal{E}^{(2)}\left(d_{21}\right)+A(\eta) \mathcal{E}^{(2)}\left(d_{32}\right)
$$

Здесь

$$
\begin{aligned}
A(\eta)= & \int_{0}^{\infty} y^{2} d y \int_{0}^{\infty} d x \ln \left\{\left(1-\frac{2 \eta^{2} x^{2} e^{-y}}{(1+\eta x)(1+2 \eta x)}\right)\left(1-\frac{2 \eta^{2} e^{-y}}{(x+\eta)(x+2 \eta)}\right)\right\} \times \\
& \times\left[\int_{0}^{\infty} y^{2} d y \int_{0}^{\infty} d x \ln \left\{\left(1-\frac{\eta^{2} x^{2} e^{-y}}{(1+\eta x)^{2}}\right)\left(1-\frac{\eta^{2} e^{-y}}{(x+\eta)^{2}}\right)\right\}\right]^{-1} .
\end{aligned}
$$

Функция $A(\eta)$ монотонно стремится к единице при $\eta \rightarrow \infty$, начиная со значения $A(0)=1.3857 \ldots$. Таким образом, вторая плоскость дает аддитивный вклад в энергию с весом $A(\eta)$. 
3.3. Четыре плоскости. Расположим плоскости в точках $d_{1}<d_{2}<d_{3}<d_{4}$. Вычтем из энергии выражение, полученное последовательным взятием пределов $d_{4} \rightarrow \infty, d_{3} \rightarrow \infty$ и $d_{2} \rightarrow \infty$. Имеем следующие выражения для энергий в сферических координатах:

$$
\begin{aligned}
\mathcal{E}_{\mathrm{TE}}^{(4)} & =\frac{1}{32 \pi^{2}} \int_{0}^{\infty} \kappa^{2} d \kappa \int_{0}^{1} d x \ln \left(1-\frac{\eta^{2} x^{2}}{(1+\eta x)^{2}}\left\{e^{-d_{21} \kappa}+e^{-d_{32} \kappa}+e^{-d_{43} \kappa}+\right.\right. \\
& \left.\left.+\frac{1-\eta x}{1+\eta x}\left(e^{-d_{31} \kappa}+e^{-d_{42} \kappa}\right)+\left(\frac{1-\eta x}{1+\eta x}\right)^{2} e^{-d_{41} \kappa}-\frac{\eta^{2} x^{2}}{(1+\eta x)^{2}} e^{-\left(d_{43}+d_{21}\right) \kappa}\right\}\right), \\
\mathcal{E}_{\mathrm{TM}}^{(4)} & =\frac{1}{32 \pi^{2}} \int_{0}^{\infty} \kappa^{2} d \kappa \int_{0}^{1} d x \ln \left(1-\frac{\eta^{2}}{(\eta+x)^{2}}\left\{e^{-d_{21} \kappa}+e^{-d_{32} \kappa}+e^{-d_{43} \kappa}+\right.\right. \\
& \left.\left.+\frac{x-\eta}{x+\eta}\left(e^{-d_{31} \kappa}+e^{-d_{42} \kappa}\right)+\left(\frac{x-\eta}{x+\eta}\right)^{2} e^{-d_{41} \kappa}-\frac{\eta^{2}}{(x+\eta)^{2}} e^{-\left(d_{43}+d_{21}\right) \kappa}\right\}\right) .
\end{aligned}
$$

Как и в случае трех плоскостей, легко показать, что в идеальном случае $\eta \rightarrow \infty$ энергия Казимира будет простой суммой трех слагаемых,

$$
\mathcal{E}^{(4)}=\mathcal{E}_{\mathrm{c}}^{(2)}\left(d_{21}\right)+\mathcal{E}_{\mathrm{c}}^{(2)}\left(d_{32}\right)+\mathcal{E}_{\mathrm{c}}^{(2)}\left(d_{43}\right),
$$

соответствующих энергиям Казимира для трех пар соседних плоскостей.

В случае малой проводимости $\eta \ll 1$ главный вклад дает ТМ-компонента:

$$
\begin{aligned}
\mathcal{E}^{(4)} & =\frac{\eta}{4 \pi^{2}} \int_{0}^{\infty} \kappa^{2} d \kappa \int_{0}^{\infty} d x \ln \left(1-\frac{1}{(1+x)^{2}}\left\{e^{-2 d_{21} \kappa}+e^{-2 d_{32} \kappa}+e^{-2 d_{43} \kappa}+\right.\right. \\
& \left.\left.+\frac{x-1}{x+1}\left(e^{-2 d_{31} \kappa}+e^{-2 d_{42} \kappa}\right)+\left(\frac{x-1}{x+1}\right)^{2} e^{-2 d_{41} \kappa}-\frac{1}{(x+1)^{2}} e^{-2\left(d_{43}+d_{21}\right) \kappa}\right\}\right) .
\end{aligned}
$$

$\mathrm{B}$ случае равных расстояний между плоскостями $d_{21}=d_{32}=d_{43}=d$ зависимость от расстояния между плоскостями имеет простой вид

$$
\mathcal{E}^{(4)}=\frac{Q^{(4)}(\eta)}{d^{3}}, \quad Q^{(4)}=Q_{\mathrm{TE}}^{(4)}+Q_{\mathrm{TM}}^{(4)},
$$

где

$$
\begin{aligned}
& Q_{\mathrm{TE}}^{(4)}=\frac{1}{32 \pi^{2}} \int_{0}^{\infty} y^{2} d y \int_{0}^{1} d x \ln \left(1-\frac{\eta^{2} x^{2} e^{-y}}{(1+\eta x)^{2}}\left\{3+\frac{2-3 \eta^{2} x^{2}}{(1+\eta x)^{2}} e^{-y}+\frac{(1-\eta x)^{2}}{(1+\eta x)^{2}} e^{-2 y}\right\}\right), \\
& Q_{\mathrm{TM}}^{(4)}=\frac{1}{32 \pi^{2}} \int_{0}^{\infty} \kappa^{2} d \kappa \int_{0}^{1} d x \ln \left(1-\frac{\eta^{2} e^{-y}}{(x+\eta)^{2}}\left\{3+\frac{2 x^{2}-3 \eta^{2}}{(x+\eta)^{2}} e^{-y}+\frac{(x-\eta)^{2}}{(x+\eta)^{2}} e^{-2 y}\right\}\right) .
\end{aligned}
$$

Для малой проводимости $\eta \ll 1$ получаем

$$
\mathcal{E}^{(4)}=-\frac{a_{4} \eta}{2 \pi d^{3}}=-\frac{a_{4} \sigma}{d^{3}},
$$

где

$$
\begin{aligned}
a_{4} & =-\frac{1}{16 \pi} \int_{0}^{\infty} y^{2} d y \int_{0}^{\infty} d x \ln \left(1-\frac{e^{-y}}{(x+1)^{2}}\left\{3+\frac{2 x^{2}-3}{(x+1)^{2}} e^{-y}+\frac{(x-1)^{2}}{(x+1)^{2}} e^{-2 y}\right\}\right)= \\
& =0.126159 \ldots .
\end{aligned}
$$

При удалении двух плоскостей от двух выбранных энергия не разделяется на простую сумму попарных энергий, а зависит от взаимного расположения удаленных плоскостей. 


\section{4. ЗАКЛЮЧЕНИЕ}

Суммируем результаты работы. Мы рассмотрели систему проводящих параллельных плоскостей, обладающих постоянной проводимостью. В случае одинакового расстояния $d$ между плоскостями энергия Казимира для системы $n$ плоскостей обратно пропорциональна $d^{3}$. Такой вывод можно сделать из простых размерных соображений. Действительно, в случае одинаковых расстояний между плоскостями в задаче имеется единственный параметр с размерностью длины $d$. Проводимость поверхностей $\sigma$ имеет размерность скорости и поэтому образует безразмерный параметр $\eta=2 \pi \sigma / c$. Таким образом, энергия системы на единицу площади плоскости имеет следующую структуру:

$$
\mathcal{E}^{(n)}=\frac{\hbar c}{d^{3}} Q_{n}(\eta)
$$

В случае малой проводимости, когда $2 \pi \sigma \ll c$, величина $Q(\eta)$ порядка $\eta$, и энергия имеет следующий вид:

$$
\mathcal{E}^{(n)} \approx-\hbar \frac{a_{n} \sigma}{d^{3}}
$$

где $a_{2}=0.0407 \ldots, a_{3}=0.0832 \ldots, a_{4}=0.1261 \ldots$. Для листов графена проводимость является универсальной константой: $\sigma=e^{2} / 4 \hbar$. В этом случае энергия не зависит от постоянной Планка и скорости света,

$$
\mathcal{E}^{(n)} \approx-\frac{a_{n} e^{2}}{4 d^{3}}
$$

Для системы $n$ идеально проводящих плоскостей, когда $\sigma \rightarrow \infty$, энергия Казимира представляет собой сумму энергий Казимира соседних пар плоскостей. Это вполне очевидно, если учесть, что условие идеальности приводит к дискретному спектру энергий между плоскостями и непрерывному спектру вне плоскостей. Конечная проводимость нарушает эту простую закономерность, и энергия становится сложной функцией, зависящей от взаимных расстояний $d_{i k}$ между парами плоскостей с любыми номерами $i$ и $k$. Для системы трех и четырех плоскостей энергия имеет вид (4) и (5) соответственно.

Благодарности. Работа авторов была частично финансирована РФФИ (грант № 13-02-00757_а).

\section{Список литературы}

[1] H. B. G. Casimir, Proc. K. Ned. Akad. Wetensc., 51 (1948), 793-795.

[2] M. Bordag, U. Mohideen, V. M. Mostepanenko, Phys. Rep., 353:1-3 (2001), 1-205, arXiv: quant-ph/0106045.

[3] K. A. Milton, J. Phys. A: Math. Gen., 37:38 (2004), R209-R277.

[4] S. Y. Buhmann, D.-G. Welsch, Prog. Quantum Electron., 31:2 (2007), 51-130.

[5] G. L. Klimchitskaya, U. Mohideen, V. M. Mostepanenko, Rev. Modern Phys., 81:4 (2009), $1827-1885$.

[6] K. A. Milton, Casimir Effect. Physical Manifestation of Zero-Point Energy, World Sci., Singapore, 2001.

[7] M. Bordag, G. Klimchitskaya, U. Mohideen, V. Mostepanenko, Advances in the Casimir Effect, International Series of Monographs on Physics, 145, Oxford Univ. Press, Oxford, 2009. 
[8] Е. М. Лифшиц, ЖЭТФ, 29:1 (1955), 94-110.

[9] И. Е. Дзялошинский, Е. М. Лифшиц, Л. П. Питаевский, УФН, 73 (1961), 381-422.

[10] Ю. С. Бараш, В. Л. Гинзбург, УФН, 116 (1975), 5-40.

[11] J.S. Dowker, R. Critchley, Phys. Rev. D, 13:12 (1976), 3224-3232.

[12] S. W. Hawking, Commun. Math. Phys., 55:2 (1977), 133-148.

[13] S. K. Blau, M. Visser, A. Wipf, Nucl. Phys. B, 310:1 (1988), 163-180.

[14] E. Elizalde, S. D. Odintsov, A. Romeo, A. A. Bytsenko, S. Zerbini, Zeta Regularization Techniques with Applications, World Sci., Singapore, 1994.

[15] M. Bordag, E. Elizalde, K. Kirsten, S. Leseduarte, Phys. Rev. D, 56:8 (1997), 4896-4904, arXiv: hep-th/9608071.

[16] Ю. С. Бараш, В. Л. Гинзбург, УФН, 143 (1984), 345-389.

[17] V. V. Nesterenko, I. G. Pirozhenko, Phys. Rev. A, 86:5 (2012), 052503, 13 pp.

[18] Н. Р. Хуснутдинов, А. Р. Хабибуллин, ТМФ, 166:1 (2011), 77-94.

[19] M. Bordag, N.R. Khusnutdinov, Phys. Rev. D, 77:8 (2008), 085026, 12 pp., arXiv: 0801.2062 .

[20] K. S. Novoselov, A. K. Geim, S. V. Morozov, D. Jiang, Y. Zhang, S. V. Dubonos, I. V. Grigorieva, A. A. Firsov, Science, 306:5696 (2004), 666-669, arXiv: cond-mat/0410550.

[21] L. A. Falkovsky, A. A. Varlamov, Eur. Phys J. B, 56:4 (2007), 281-284.

[22] A. B. Kuzmenko, E. van Heumen, F. Carbone, D. van der Marel, Phys. Rev. Lett., 100:11 (2008), 117401, 4 pp.

[23] R. R. Nair, P. Blake, A. N. Grigorenko, K.S. Novoselov, T. J. Booth, T. Stauber, N. M. R. Peres, A. K. Geim, Science, 320:5881 (2008), 1308-1308.

[24] I. V. Fialkovsky, V. N. Marachevsky, D. V. Vassilevich, Phys. Rev. B, 84:3 (2011), 035446, 10 pp., arXiv: 1102.1757.

[25] V. N. Marachevsky, J. Phys. A: Math. Theor., 45:37 (2012), 374021, 17 pp.

[26] V. N. Marachevsky, Internat. J. Modern Phys., 14 (2012), 435-444, arXiv: 1111.3612.

[27] D. Drosdoff, L. M. Woods, Phys. Rev. B, 82:15 (2010), 155459, 10 pp.

[28] N. Khusnutdinov, D. Drosdoff, L. M. Woods, Phys. Rev. D, 89 (2014), 085033, 10 pp., arXiv: 1404.2532 .

Поступила в редакцию 4.09.2014 